\title{
Autoimmune Hepatitis Triggered By Hepatitis A Viral Infection (A case report )
}

\author{
Talib Shafaat .Hussain*, Gopanpallikar Aniruddha**, Wawhal Mahendra***, \\ Quadri Umar****, Sainani Rahul *****,Deshpande Shreyas****** \\ * Professor \& Head, ** Consulting Gastroenterologist , ***Associate Professor, ****Lecturer, ***** \\ Chief Resident, ******Senior Resident \\ Department Of Medicine, Mahatma Gandhi Missions Medical College, Hospital \& Gopanpallikar \\ Gastroenterology Institute , Aurangabad.
}

\begin{abstract}
Hepatitis A virus is an infectious agent known to trigger autoimmune hepatitis ( AIH ). We present a case in a 45 year old woman with interstitial lung disease \& chronic active hepatitis. Diagnosis of hepatitis A virus was attributed on viral serological tests \& autoimmune hepatitis $(A I H)$ in accordance with international autoimmune hepatitis group system ${ }^{I}$. She is in remission with steroid therapy. The case we present is unusual with paucity observed in the world literature.
\end{abstract}

Key words: Acute hepatitis A , Autoimmune hepatitis , HLA, Interstitial lung disease, International criteria for AIH.

\section{Introduction :}

We present a case of 45 yrs old woman who has had jaundice as the chief complaint 6 months earlier . She developed nausea, aversion to food \& dry cough with frequent motions post meal . Tests on patient indicated chronic active hepatitis with interstitial lung disease. Diagnosis of hepatitis A virus was attributed on negative hepatitis B surface antigen, negative hepatitis $\mathrm{C}$ virus antibody, \& positive anti hepatitis A virus antibody ( HAV-T ). Possibility of Autoimmune Hepatitis ( AIH ) was considered as patient had jaundice related to recent past hepatitis A infection, Interstitial lung disease, mildly deranged thyroid functions, Elevation of Antinuclear antibodies \& abnormal AST , ALT levels . Liver biopsy was confirmative of chronic active hepatitis . Serological, immunological \& histopathological parameters were in confirmation for modified international criteria for AIH . Steroid therapy was initiated with fruitful outcome (noticed 6 weeks later ). The present case was thus considered to represent AIH triggered by Hepatitis A viral infection .

\section{Case Description:}

Patients history dates back to 2 yrs when she had dry cough \& onset of gradually progressive dyspnea ( grade $1-3$ ). She was seen by chest physician for the said problems a year ago. Interstitial lung disease was diagnosed clinically \& on CT imaging ( Fig 1 ) for which she received steroids 40mg/day in tapering doses for 6 months . Cough receded, however she had mild cushingoid features \& hypertension, treated with Losartan 50 $\mathrm{mg} /$ day which she continued for 2 months. Past medical history further revealed occurrence of jaundice $20 \mathrm{yrs}$ back for 1 month for which she received supportive therapy. She further had developed jaundice 6 months back $\&$ received supportive therapy. 3 months prior to the present hospitalization she subsequently developed poor appetite, nausea \& aversion to food, loose stools as \& when on taking meals . For the said problem, gastro endoscopy done revealed only hyperaemic stomach. She was kept on supportive treatment with antacids, vitamins \& minerals . As her symptoms persisted she seeked hospitalization for further investigations \& management.

In the hospital, examination revealed middle aged lady who had no prior history of alcohol or substance abuse \& family history of liver disease. Non tender hepatomegaly of 4.5 inches below costal margin was noted on her physical examination. Velcro rales at both lung bases noted . Other systemic examination was unrevealing . Roentgenography chest revealed increased reticulonodular shadows at both bases with marked elevation of right dome of diaphragm ( Fig.2 ). Sonography abdomen showed moderate hepatomegaly with fatty liver, no ascites . Her laboratory findings were as follows : $\mathrm{Hb}: 9.6 \mathrm{gm} \%$, TLC $-9180 /$ cumm , P : $85 \%$, L : $12 \%, \mathrm{E}: 01 \%, \mathrm{M}: 2 \%$, platelets were adequate $-2,61,000 / \mathrm{cumm}$, ESR : $55 \mathrm{~mm} / \mathrm{hr}$, Total Bilirubin 0.94 $\mathrm{mg} / \mathrm{dl}$, Direct Bilirubin $0.29 \mathrm{mg} / \mathrm{dl}$, AST $102 \mathrm{U} / \mathrm{L}$ ( N : 0-31 ), ALT 61.2 U/L ( N : 0-31 ), Serum Alkaline Phosphatase 196.4 U/L ( N : 42-98), PT $13.5 \mathrm{sec}$, INR 1.23, S Albumin $2.4 \mathrm{gm} / \mathrm{ml}$, S Globulin $5.1 \mathrm{gm} / \mathrm{ml}$, TSH $4.76 \mathrm{uIU} / \mathrm{ml}$ ( N - 0.4-4 ), Lipid profile was within normal limit, Serum anti HAV Total was positive, Serum anti HAV IgM was negative. $\mathrm{HBsAg}$, anti $\mathrm{HBs}, \mathrm{HBeAg}$, anti $\mathrm{HBeAg}$, anti $\mathrm{HBc} \mathrm{IgM}$, anti $\mathrm{HCV}$ were negative. Her antinuclear antibody \& rheumatoid factor was positive while anti smooth muscle antibody \& anti liver kidney microsomal 1 antibody tests were negative. Serum ferritin levels were within normal limits . HLA 
assessment conducted after admission revealed : B44(12) ; B51 ( 5 ) ; DR4 ; DR9. Liver histopathology revealed fatty degeneration in hepatocytes, portal tract widening with mononuclear cell infiltration \& piecemeal necrosis with early fibrosis. ( Fig 3 ). The patient had a score of 22 according to the international autoimmune hepatitis group system. She was provided with prednisolone $40 \mathrm{mg} / \mathrm{day}$ ( in tapering doses ) \& observed to be in remission with the therapy 8 weeks later .

\section{Discussion :}

patitis A virus ( HAV) is an enteric transmitting virus which is the most common cause of acute viral hepatitis worldwide ${ }^{2}$. In the present case though HAV ( IgM ) was negative, however competitive enzyme immunoassay for anti Hepatitis A virus Total ( HAVT ) was significantly positive as patient has had jaundice 6 months earlier . IgM antibodies are known to disappear after the acute infection within 5-6 months while IgG antibodies persists lifelong. Hepatitis A virus is also incriminated as a triggering cause for autoimmune hepatitis ${ }^{3,4,5}$. AIH is a rare disease with unknown etiology which causes chronic necro inflammatory changes in the liver . Hepatitis $\mathrm{C}$ has been associated with AIH more than any other hepatitis viruses ${ }^{6}$. Viral proteins of viruses may be similar to the amino acid chain of different autoantigens in the liver . Cross immune reactions may also be responsible for the damage in the liver tissue. AIH appears to be induced by antibody dependent cell mediated cytotoxicity which involves both antibody mediated \& cellular immunity against specific liver antigens as hepatocyte membranes ${ }^{7}$. There are several genetic factors including human lymphocyte antigen ( HLA ) types which are related to AIH such as DR3, DR $4^{8}$. Hepatitis A as a triggering factor was considered in the case as Hepatitis HAVT was positive \& Hepatitis B surface antigen, core antigen, HBe antibodies, HCV antibodies were negative in the case . Liver histopathology revealed fatty degeneration in hepatocytes; portal tract widening with mononuclear cell infiltrate \& areas of piecemeal necrosis with early fibrosis. The features consistent with the diagnosis of chronic active hepatitis . ANA was positive however ASMA, AMA \& anti LKM1 antibodies were negative. HLA analysis of the case revealed B44(12) ; B51 ( 5 ) ; DR4 ; DR9.

Many autoimmune conditions like thyroiditis, ulcerative colitis, celiac sprue, mixed connective tissue disorder, interstitial lung disease, Autoimmune haemolytic anaemia are associated with $\mathrm{AIH}^{9}$

Histopathology scoring for autoimmune hepatitis in the case revealed a score of 12 , the value is in consistence with the definite diagnosis of $\mathrm{AIH}^{4}$. In the present case there has not been enough observed reasons to speculate any other assumptive etiological clue. HAV infection is probably one which is well documented in the case out of several unknown triggers that may induce autoimmune hepatitis . This patient on other hand had interstitial lung disease long before the HAV infection ( as jaundice was noted couple of months later of onset of ILD ). A speculation in the case could be logical if assumed that AIH existed earlier along with ILD before the HAV infection which has further worsened the liver as evident in the present case from clinical, serological \& immunological \& imaging studies .It is further speculated that this patient under discussion has had revealed isolated antiHBc positivity with neither HBsAg nor AntiHBs which may indicate either :

1 ) Case with many years after recovery from acute hepatitis B when AntiHBs has fallen to undetectable levels or

2 ) Case presentation after many years of chronic HBV infection when the HBsAg has been cleared which occurs in less than $1 \%$ of the carriers ${ }^{10}$.

It is therefore proposed that the present case might have had perhaps liver disease of hepatitis B antigen years ago with HBsAg \& AntiHBs now have fallen to undectable levels \& concurrent hepatitis A infection ( AntiHAV positivity ) did the damage further \& resulted in acute autoimmune hepatitis . It is for the said reason vaccination of $\mathrm{HAV}$ is recommended worldwide for all patients with chronic liver disease including hepatitis $\mathrm{C}$ patients to prevent decompensation due to HAV superinfections ${ }^{11}$. However Bary \& Smith 2007 described the first ever case of AIH in association with vaccination against HAV after 10 days post vaccination ${ }^{\mathbf{1 2}}$. There is paucity in literature of HAV with AIH and / or dual edged sword infections of HBsAg with HAV as old \& new infections with AIH inter-relationship. In our case, there are strong evidences favouring Hepatitis A virus ( $\mathrm{HAV}$ ) as a triggering factor leading to AIH .

\section{References :}

[1]. Gleeson D, Heneghan M. British Society of gastroenterology ( BSG ) guidelines for management of autoimmune hepatitis. Gut. 2011;60:1611-29.

[2]. Tosun S, Ertan P, Kasirga E, Atman U. Changes in seroprevalence of hepatitis A in children and adolescents in Manisa, Turkey. Pediatr Int 2004;46:669-72

[3]. Singh G, Palaniappan S, Rotimi O, Hamlin PJ. Autoimmune hepatitis trigerred by hepatitis A. Gut. 2007;56:304

[4]. Tabak F, Ozdemir F, Tabak O, Erer B, Tahan V, Ozaras R. Autoimmune hepatitis induced by the prolonged hepatits A virus infection. Annals of Hepatology 2008;7(2): 177-79.

[5]. Tanaka H, Tujioka H, Ueda H, Hamagami H, Kida Y, Ichinose M. Autoimmune hepatitis triggered acute hepatitis A. World J Gastroenterol 2005;11(38):6069-71.

[6]. Strassburg CP, Vogel A, Manns MP. Autommunity and hepatitis C. Autoimmun Rev 2003;2:322-31. 
[7]. Eggink HF, Houthoff HJ, Huitema S, Gips CH, Poppema S. Cellular and humoral immune reactions in chronic liver disease.I.Lymphocyte subsets in liver biopsies of patients with untreated idiopathic autoimmune hepatitis, chronic active hepatitis B and primary biliary cirrhosis. Clin Exp Immunol 1982;50:17-24

[8]. Czaja AJ, Carpenter HA, Santrach PJ, Moore SB. DR human leukocyte antigen and disease severity in chronic hepatitis C. J Hepatol 1996;24:666-73

[9]. 1 Mcphee SJ, Papadauis MA. Autoimmune hepatitis in 2010 current medical diagnosis and treatment. 49 ${ }^{\text {th }}$ ed. Tata McGrawHill;New Delhi. 2010. p. 612-14.

[10]. Wilks D, Ferrington M, Rubenstein D. Hepatitis in The infectious diseases manual $2^{\text {nd }}$ ed, Blackwell publishing; 2003. p. 70-6.

[11]. Kallinowski B, Jilg W, Buccholz L. Stremmel W, Engler S. Immunogenecity of an accelerated vaccination regime with a combined hepatitis a/b vaccine in patients with chronic hepatitis C. Z Gastroenterol 2003;41:983-90

[12]. Berry PA, Laing GS. Hepatitis A vaccine associated with autoimmune hepatitis. World J Gastroenterol 2007;13(15):2238-39.

Legends to figures :

Fig 1 : HRCT chest showing bilateral basal pneumonia with ground glass appearance \& reticular shadows suggestive of interstitial lung disease .

Fig 2 : XRAY chest showing reticulonodular shadows at both lung bases with elevation of right dome of diaphragm.

Fig 3 : Liver histopathology $\mathrm{H} \& \mathrm{E}$ stain ( 100x ) revealing fatty degeneration in hepatocytes with portal tract widening, mononuclear cell infiltration, piecemeal necrosis \& early fibrosis .

\section{Fig 1 :}

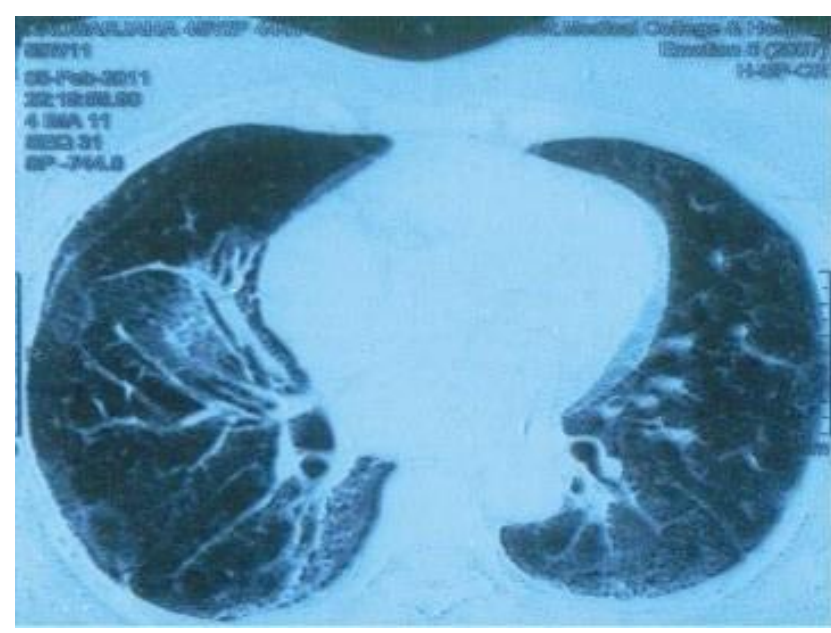

Fig 2 :

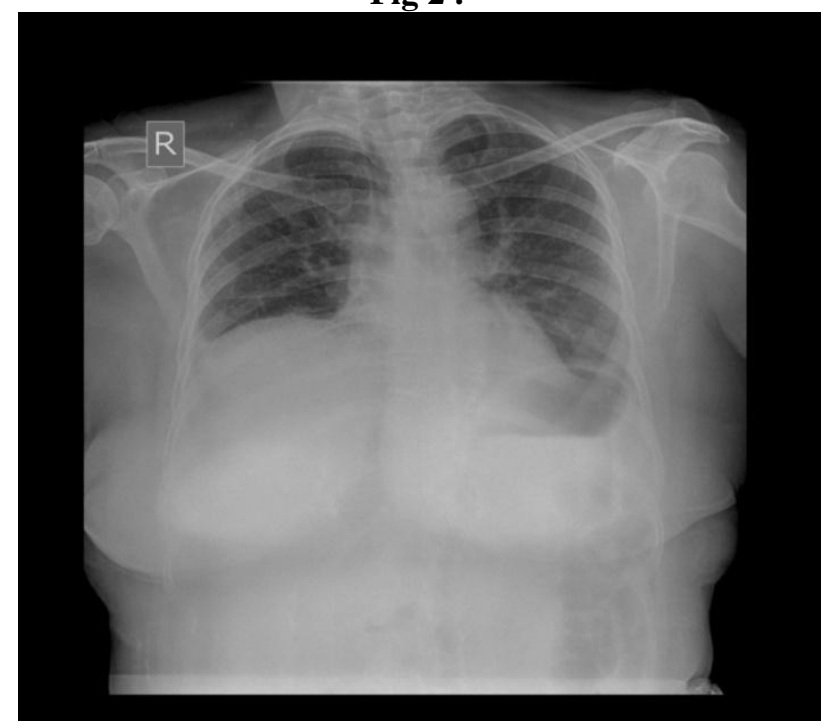


Fig 3 :

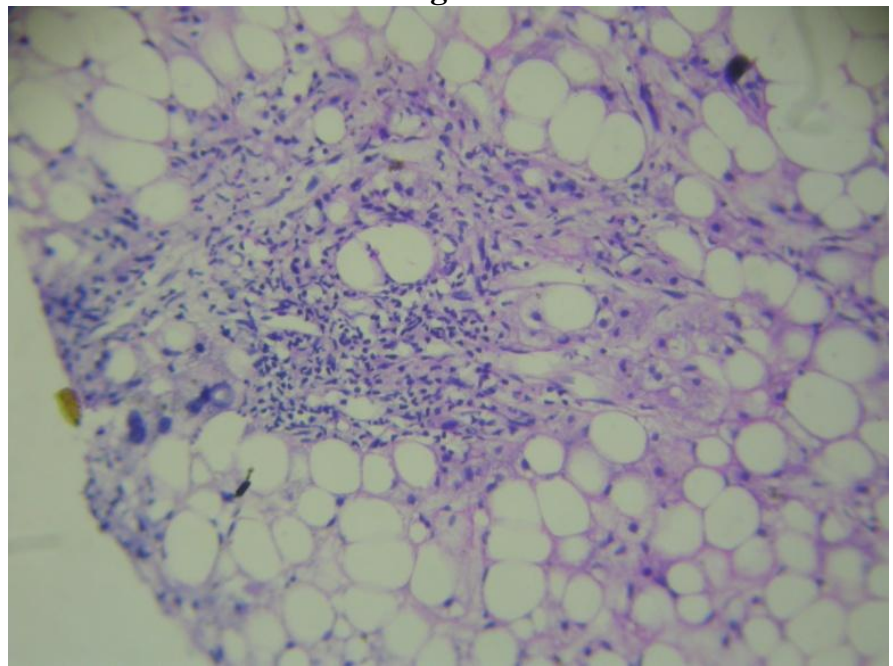

